\title{
Commentary on Trevor and Huron, "Are Humoresques Humorous? On the Acoustic Similarity Between Laughter and Staccato"
}

\author{
CHRISTOPHER S. LEE[1] \\ Goldsmiths College, University of London, UK
}

\begin{abstract}
In this commentary, several problematic aspects of Trevor and Huron's study are discussed and suggestions made for improving the experimental design.
\end{abstract}

Submitted 2018 February 8; accepted 2018 August 2.

KEYWORDS: Music and laughter, staccato, laughter types, rate, emotional contagion

TREVOR and Huron's hypothesis that composers emulate the sound of human laughter through using isochronous staccato passages when writing humorous music is novel and interesting, as it posits an analogue between vocal and musical expression which is distinct from those based on the physiological state cues involving speech rate, fundamental frequency (F0), and voice quality that have been the focus of most research to date (Juslin \& Laukka, 2003; Scherer, 1986). As the authors themselves admit, however, the evidence they present in support of the hypothesis is not particularly strong. In part one of the study, where participants were asked to adjust tone sequences (and a vocal sequence) to emulate laughter, results showed that the mean rates chosen were slower than reference values taken from three studies of actual human laughter. In part two, a corpus analysis, compositions with humorous titles were not found to contain more isochronous staccato passages than matched control compositions, contrary to their hypothesis. In this commentary, I highlight some weaknesses in the rationale and methodology of the study, which may help explain the failure to obtain more convincing results, and suggest some changes that might produce more clear-cut findings.

There are two issues with the experiment in part one of the study. First, it is assumed that, at least as far as rate and duty cycle are concerned, human laughter can be regarded as a unitary phenomenon, and that it therefore makes sense to compare overall means. However, Szameitat et al. (2009), in a study of professional actor portrayals of four common laughter types (associated with joy, tickling, schadenfreude, and taunting), found that different laughter types displayed distinct rates, with the rate parameter modulated differently across the types according to the sex of the speaker: the slowest rates were for male schadenfreude laughter $(3.77 \mathrm{~Hz})$ and female taunting laughter $(4.07 \mathrm{~Hz})$, whereas the fastest rates were for male and female tickling laughter $(4.87$ and $4.60 \mathrm{~Hz}$ respectively). It is possible, therefore, that the rates obtained in the current experiment reflect the rates of slower laughter types rather than, as suggested by the authors, the typically slower rates of fake (non-spontaneous) laughter in general; however, this latter hypothesis is still highly plausible, given that the unvarying parameters of the experimental sequences might have resulted in sequences that sound fake, as the authors themselves observe (see Kipper \& Todt, 2001). One way of establishing whether there is an influence of imagined laughter types would be to modify the experiment to distinguish between the fastest type (i.e. tickling laughter), and other types. This might be achieved by using the characteristically higher mean F0 of tickling laughter as a distinguishing cue, and setting up a high-pitched and low-pitched condition, with the two conditions containing appropriately pitched (female) laughter syllables and similarly-pitched instrumental sound sequences, to assess whether the conditions yield appropriately distinct rates. Such a modification would also yield valuable information on the likely importance of both pitch and the interaction between pitch and rate as cues in musical portrayals of laughter.

The second issue concerns the adequacy of the experimental paradigm. While the experiment yields clear answers to the two specific hypotheses tested, it does not fully address the broader question of whether laughter-like sounds can be produced with musical instruments, because it excludes likely 
important parameters (e.g., pitch) from consideration, and most fundamentally does not address the question of the extent to which the adjusted sequences sound laughter-like. As a result, the musical relevance of the experimental findings is unclear. One way of remedying this problem would be to include an evaluation task, possibly with a separate group of participants, in which the adjusted sequences were judged in terms of how laughter-like they sounded, alongside real musical sequences overtly portraying laughter (e.g., the operatic excerpts investigated in Provine, 2000, 2008), and/or actual human laughter as a reference. A more ambitious follow-up study could also address the question of whether musical sequences emulating laughter actually evoke amusement/laughter in listeners, perhaps via a process of emotional contagion (Davies, 2013; Juslin \& Västfjäll, 2008), using self-report measures as well as physiological measures of facial activity indicative of amusement (see Wolf, 2015, for a review of techniques for measuring facial emotion, and illustrative studies in Lundqvist, Carlsson, Hilmersson, \& Juslin, 2009, and Lynch \& Trivers, 2012). If laughter-like musical sequences do evoke amusement in listeners, then this would constitute an important reason as to why they might be more common in compositions with an explicitly humorous character.

Turning now to the corpus study, there are two methodological issues, one concerning the specific hypotheses tested, and the other referring to the analysis. Regarding the hypotheses, the problem is that they are too general to yield an adequate answer to the question of whether laughter-like musical passages are more common in humorously-titled compositions. The first hypothesis (H3) embodies a claim merely about staccato sequences in general, while the second hypothesis (H4), though focused on isochronous staccato sequences and hence more relevantly specific, has nothing to say about the crucial factor of event rate. If there is no reason to expect staccato sequences that are not laughter-like to be more common in humorously-titled compositions, the net result is likely to be a loss of statistical power and/or a result that is difficult to interpret (as in this case, with confirmation of $\mathrm{H} 3$ but not $\mathrm{H} 4$ ). How then might event rate be considered? One way this could be achieved would be to exclude all isochronous sequences with a periodicity at or above the level of the tactus, on the basis that the aggregate mean preferred rate of $3.65 \mathrm{~Hz}$ in the experiment is equivalent to a tactus rate of $219 \mathrm{bpm}$; this is already faster than typical rates found in classical compositions. The proposed condition is crude, but it would probably exclude very few sequences that would be perceived as laughter-like, and would likely result in a more sensitive test.

Regarding the analysis, there are two problems with the adopted procedure of simply comparing the numbers of compositions containing at least one putatively laughter-like passage. First, it does not consider possible differences between the two sets in the prevalence of such passages within compositions, even though the humorously-titled compositions might be expected to contain more laughter-like passages than the control compositions. Second, it is affected by the confound of length, since the humorously-titled compositions are longer than their control counterparts, and longer compositions are statistically more likely to contain passages meeting any given criteria than shorter compositions, as the authors themselves note. It would therefore be preferable to perform a full analysis of the two sets of compositions and for each composition calculate the percentage of bars containing laughter-like passages, or alternatively to adopt a less taxing procedure originally proposed by the second author in a study of syncopation in American popular music (Huron \& Ommen, 2006), and measure the percentage of randomly sampled bars containing laughter-like passages. This procedure is both sensitive to prevalence and avoids the length confound, since the likelihood that a randomly sampled bar contains such a passage increases with their prevalence, and does not increase with the length of the composition.

In conclusion, Trevor and Huron present an interesting and provocative thesis, but provide only limited evidence in support. However, a study incorporating the suggestions proposed in this commentary could yield more decisive results.

\section{ACKNOWLEDGEMENTS}

This article was copyedited by Scott Bannister and layout edited by Kelly Jakubowski.

\section{NOTES}

[1] Correspondence can be addressed to christopher.lee@gold.ac.uk. 


\section{REFERENCES}

Davies, S. (2013). Music-to-listener emotional contagion. In: T. Cochrane, B. Fantini, \& K. Scherer (Eds.), The Emotional Power of Music. Oxford: Oxford University Press. https://doi.org/10.1093/acprof:oso/ 9780199654888.003 .0013

Huron, D., \& Ommen, A. (2006). An empirical study of syncopation in American popular music, 18901939. Music Theory Spectrum, 28(2), 211-232. https://doi.org/10.1525/mts.2006.28.2.211

Juslin, P. N., \& Laukka, P. (2003). Communication of emotions in vocal expression and music performance: Different channels, same code? Psychological Bulletin, 129(5), 770-814. https://doi.org/10.1037/0033-2909.129.5.770

Juslin, P. N., \& Västfjäll, D. (2008). Emotional responses to music: The need to consider underlying mechanisms. Behavioral and Brain Sciences, 31(5), 559-575. https://doi.org/10.1017/S0140525X08005293

Kipper, S., \& Todt, D. (2001). Variation of sound parameters affects the evaluation of human laughter. Behaviour, 138, 1161-1178. https://doi.org/10.1163/156853901753287181

Lundqvist, L.-O., Carlsson, F., Hilmersson, P. \& Juslin, P. N. (2009). Emotional responses to music: Experience, expression, and physiology. Psychology of Music, 37(1), 61-90. https://doi.org/10.1177/0305735607086048

Lynch, R. F., \& Trivers, R. L. (2012). Self-deception inhibits laughter. Personality and Individual Differences, 53, 491-495. https://doi.org/10.1016/j.paid.2012.02.017

Provine, R. R. (2000). Laughter: A Scientific Investigation. New York: Viking Penguin.

Provine, R. R. (2008). Notation and expression of emotion in operatic laughter. Behavioral and Brain Sciences, 31, 591-592. https://doi.org/10.1017/S0140525X08005463

Scherer, K. R. (1986). Vocal affect expression: A review and a model for future research. Psychological Bulletin, 99, 143-165. https://doi.org/10.1037/0033-2909.99.2.143

Szameitat, D. P., Alter, K., Szameitat, A. J., Wildgruber, D., Sterr, A., \& Darwin, C. J. (2009). Acoustic profiles of distinct emotional expressions in laughter. Journal of the Acoustical Society of America, 126(1), 354-366. https://doi.org/10.1121/1.3139899

Wolf, K. (2015). Measuring facial expression of emotion. Dialogues in Clinical Neuroscience, 17(4), 457462. 